\title{
In-fiber Silicon Microspheres
}

\author{
L.M. Xiao ${ }^{1,2 *}$, N. Healy ${ }^{2}$, Z. Webber ${ }^{3}$, T. Hawkins ${ }^{4}$, M. Jones ${ }^{4}$, J. Ballato ${ }^{4}$, U. Gibson ${ }^{5}$, and A. C. Peacock ${ }^{2 *}$ \\ ${ }^{1}$ Key Laboratory for Micro and Nano Photonic Structures (Ministry of Education), Department of Optical Science and Engineering, Fudan \\ University, Shanghai, 200433, People's Republic of China \\ ${ }^{2}$ Optoelectronics Research Centre, University of Southampton, Southampton, SO17 1BJ, UK \\ ${ }^{3}$ Department of Physics and Astronomy, University of Southampton, Southampton, SO17 1BJ, UK \\ ${ }^{4}$ The Center for Optical Materials Science and Engineering, Technologies (COMSET), Department of Material Science and Engineering, \\ Clemson, SC, 29634, USA \\ ${ }^{5}$ Department of Physics, Norwegian University of Science and Technology, 7491 Trondheim, Norway \\ Corresponding authors: liminxiao@fudan.edu.cn, acp@orc.soton.ac.uk
}

\begin{abstract}
We have proposed and demonstrated an approach to fabricate integrated in-fiber silicon microspheres, which have nearly atomically smooth surface roughness $\sim 0.16 \mathrm{~nm}$. The hybrid structure forms a cavity with high temperature sensitivity $\sim 80 \mathrm{pm} /{ }^{\circ} \mathrm{C}$.

OCIS codes: (230.3120) Integrated optics devices; (160.6000) Semiconductor Materials.
\end{abstract}

\section{Introduction}

Silicon photonics has developed rapidly over the last decade and many devices have been successfully realized such as photodetectors, optical modulators, filters, switches and microresonators. Recently, silicon microspheres have become one of the key functional blocks and received significant attention for wide ranging applications, spanning photonics, biology and green energy [1-2]. The fabrication of high quality silicon microspheres and their integration into devices are significantly important for photonics applications. Different approaches have been developed to fabricate silicon microspheres such as the chemical vapor deposition [3], solidification of molten silicon [4], laser heating of silicon particles in a liquid medium [5], and laser reformation of silicon [6]. These approaches have produced silicon microspheres with different sizes and functionalities. However, each isolated silicon microsphere produced by these methods is not convenient to be integrated for optoelectronics applications. Unlike silica microspheres which could be melted and integrated at the end of optical fibers, these silicon microspheres are generally attached to an optical fiber stem by ultraviolet glue, which is not reliable. Furthermore, coupling light in and out through the microspheres also requires very precise positioning and alignment [7]. A new approach to produce high quality silicon microspheres that are easily integrated with standard fibers is required to advance their photonic applications.

Silicon optical fibers (SOFs) [8] represent a new field of silicon photonics and have recently been used as a precursor for the fabrication of silicon microspheres. The microspheres are typically fabricated by tapering the SOFs, which produces a series of spheres with micro-scale gaps, rendering each sphere difficult to isolate and integrate for photonic devices [2]. By melting a silicon micro-rod etched from a SOF [9], Lin et al. demonstrated a silicon microsphere at the end of the SOF, however, the microsphere fabricated by the $\mathrm{CO}_{2}$ laser melting process in air will be partly oxidized which may decrease its quality. Here we propose and demonstrate a unique arc discharge reshaping approach to fabricate and integrate in-fiber silicon microspheres. Our approach not only simplifies the fabrication and integration of individual silicon microspheres, but also produces spheres with improved surface quality, roughness values up to $0.16 \mathrm{~nm}$, which is close to an atomically smooth surface. We have also characterized the properties of these silicon microspheres and will discuss their potential applications.

\section{Fabrication}

The SOFs were fabricated by a thermal drawing procedure [8], as shown in Fig. 1(a). Using these SOFs we developed a new approach to fabricate in-fiber silicon microspheres with a fusion splicer (Fig. 1(b)). As the melting point of silicon $\left(1414{ }^{\circ} \mathrm{C}\right)$ is much lower than that of silica, a temperature range exists where the silicon will be molten whilst the silica is only softened, which can be controlled via the energy of arc discharge. If the heating energy is high enough, the molten silicon cylinder will finally break into a sphere to reduce surface energy. Here we could slow down the break-up process and continue reshaping the silicon core by repeated arc discharge method [10] as shown in Fig. 2(a-d), which corresponds to arc discharges 5 times, 7 times, 8 times and 10 times, respectively, using an Ericson splicer with optimized parameters.

To integrate an in-fiber silicon microsphere at the top of a conventional single mode fiber (SMF), we have developed a 3 step approach. Firstly, a SOF with a polycrystalline core was spliced to a SMF to avoid overheating. Then the SOF was cleaved using a $\mathrm{CO}_{2}$ laser so that only a short section of the SOF remains attached to the end of 
the SMF (Fig. 2(e)). Finally the SOF segment was carefully heated by repeated arc discharges to produce the microsphere. During the process, the melted silicon cylindrical-core forms a microsphere and becomes entirely surrounded by the silica cladding. The silicon microsphere diameter in Fig. 2(f) is $27.3 \mu \mathrm{m}$.
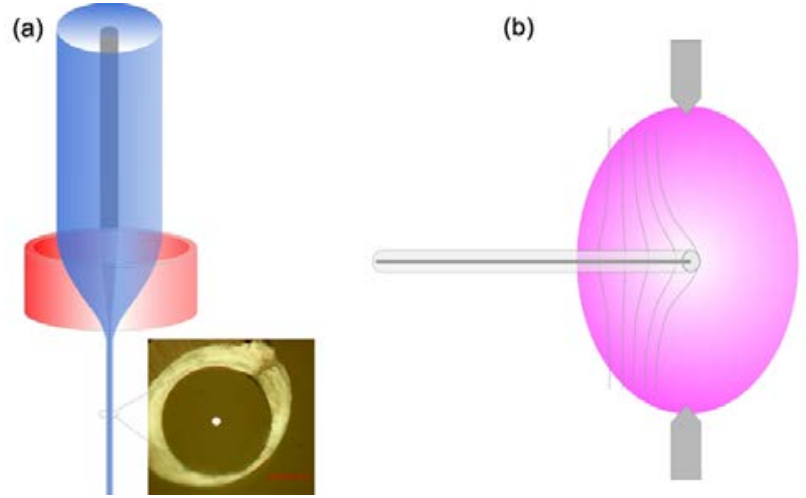

Fig. 1 Schematic images. (a) A silicon-in-silica perform is thermally drawn into a SOF, the inset is the optical micrograph of a SOF crosssection with $9.5 \mu \mathrm{m}$ diameter core (scale bar, $50 \mu \mathrm{m}$ ). (b) The SOF being heated by arc discharges of a fusion splicer.
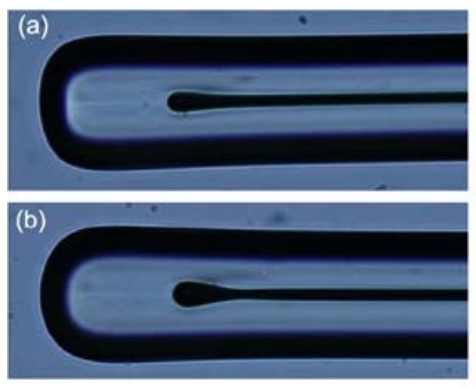
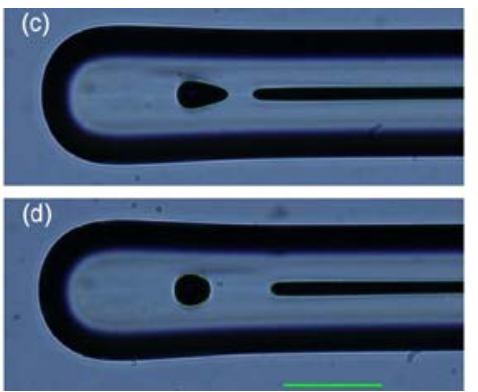

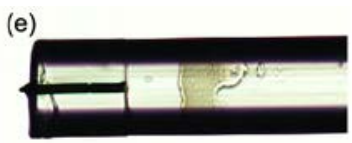

(f)

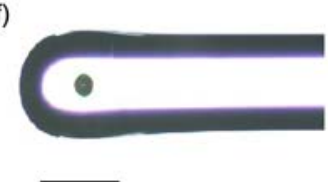

Fig. 2 Optical images of the silicon core melting process with repeated arc discharges (a, b, c, d) (scale bar, $100 \mu \mathrm{m}$ ). (e) The SOF (left) was spliced to the SMF and cleaved. (f) The silicon microsphere in silica was integrated at the top of the SMF (scale bar, $100 \mu \mathrm{m}$ ).

\section{Characterization and applications}

The reshaped silicon microsphere in Fig. 2(b) was etched using hydrofluoric acid and cleaned as shown in Fig. 3(a). A Zemetrics ZeScope three-dimensional optical profiler was used to measure the surface of the etched cylinder silicon core and the reshaped silicon microsphere part. We found that the roughness of the cylinder silicon core which was produced by thermal drawing is $\sim 0.5 \mathrm{~nm}$ (Fig. 3(b)). However, the surface roughness of the reshaped silicon microsphere is $\sim 0.16 \mathrm{~nm}$ (Fig. 3(c)). We believe that the re-melting silicon which is cladded by softened silica using our repeated arc discharge approach has improved its surface roughness. The microsphere produced in this way has a nearly atomically smooth surface and is naturally supported by the SOF, which will benefit whispering gallery mode applications of pure silicon microspheres.
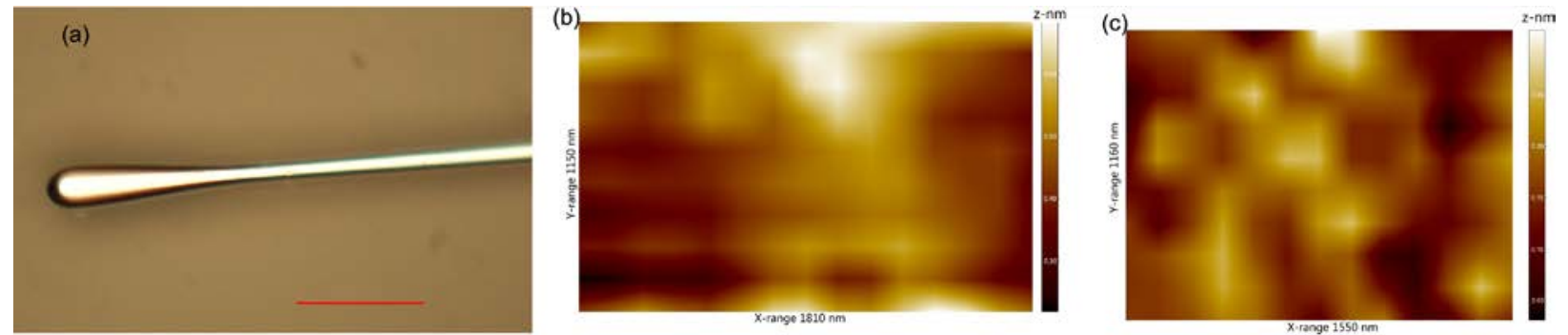

Fig. 3 (a) An etched silicon microsphere at the top of an etched silicon micro-rod (scale bar, $100 \mu \mathrm{m}$ ). (b) Surface roughness of the etched silicon micro-rod and (c) surface roughness of the etched silicon microsphere. 
To characterize the temperature response of the in-fiber microsphere in Fig. 2(f), we used a reflective set-up similar to that in Ref. [11]. Fig. 4(a) shows the measured spectrum, with a high interference fringe visibility of $\sim 25$ $\mathrm{dB}$. The fringe spacing of $12.1 \mathrm{~nm}$ matches the silicon micro-cavity length. Fig. 4 (b) shows the temperature dependence of the resonant wavelength dips over the range 30-120 ${ }^{\circ} \mathrm{C}$, and measurements have been conducted up to $750{ }^{\circ} \mathrm{C}$ without damage to the device. From the temperature tuning we can determine that the sensitivity is $\sim 80$ $\mathrm{pm} /{ }^{\circ} \mathrm{C}$, which is $\sim 40$ times more sensitive than the air-gap microcavity approach [11]. Thus the integrated silicon microspheres at the top of the SMF could act as miniature temperature sensors with high sensitivity and wide measurement range.
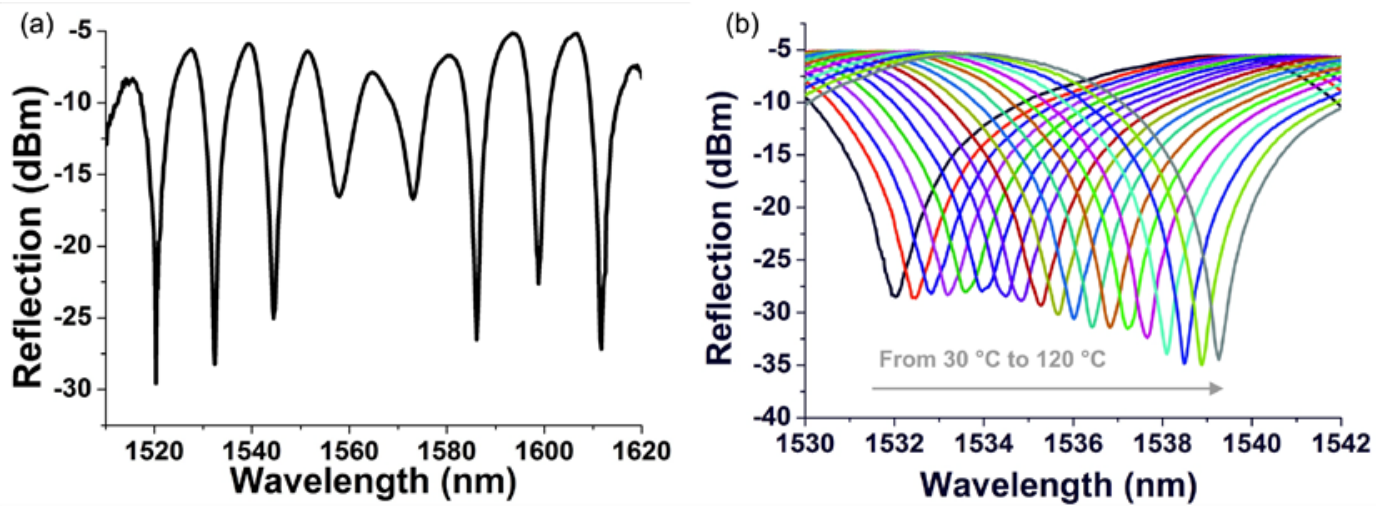

Fig. 4 (a) The interference fringes of the silicon microsphere. (b) The wavelength dip of the interference fringe at 1532nm is shifted with the increase of the temperature from $30^{\circ} \mathrm{C}$ to $120^{\circ} \mathrm{C}$ with a step of $5^{\circ} \mathrm{C}$.

\section{Conclusions}

In conclusion, we have proposed and demonstrated a new approach to fabricate and integrate silicon microspheres into conventional SMFs. Our approach has improved the surface quality of re-shaped silicon microspheres up to a nearly atomically smooth surface of $\sim 0.16 \mathrm{~nm}$. The integrated hybrid silicon microsphere cavity is sensitive to the temperature variation. This in-fiber crystalline silicon microsphere represents a new fiber-based ultracompact microsphere platform, and the high quality integrated silicon microspheres could offer opportunities in minimized fiber sensing devices and whispering gallery mode related applications.

\section{References}

[1] J. J. Kaufman, G. Tao, S. Shabahang, E. H. Banaei, D. S.Deng, X. Liang, S. G. Johnson, Y. Fink, A. F. Abouraddy, "Structured spheres generated by an in-fibre fluid instability,” Nature 487, 463-467 (2012).

[2] A. Gumennik, L. Wei, G. Lestoquoy, A. M. Stolyarov, X. Jia, P. H. Rekemeyer, M. J. Smith, X. Liang, B. J. B. Grena, S. G. Johnson, S. Gradečak, A. F. Abouraddy, J. D. Joannopoulos and Y. Fink "Silicon-in-silica spheres via axial thermal gradient in-fibre capillary instabilities," Nat. Comm., 4, 2216-2220 (2013).

[3] R. Fenollosa, F. Ramiro-Manzano, M. Tymczenko and F. Meseguer, "Porous silicon microspheres: synthesis, characterization and application to photonic microcavities,” J. Mater. Chem., 20, 5210-5214 (2010).

[4] W. R. McKee, “Development of the spherical silicon solar cell,” IEEE Trans. Comp., Hybrids, Manufact. Technol.,CHMT-5, 336-341 (1982).

[5] X. Li, A. Pyatenko, Y. Shimizu, H. Wang, K. Koga, and N. Koshizaki. 2011. "Fabrication of crystalline silicon spheres by selective laser heating in liquid medium,” Langmuir 27, 5076-5080 (2011).

[6] S. C. Hung, S. C. Shiu, C. H. Chao, C. F. Lina, "Fabrication of crystalline Si spheres with atomic-scale surface smoothness using homogenized KrF excimer laser reformation system,” J. Vac. Sci. Technol. B 27, 1156-1160 (2009).

[7] E. Xifre-Perez, J. Domenech, R. Fenollosa, P. Munoz, J. Capmany, and F. Meseguer, “All silicon waveguide spherical microcavity coupler device,” Opt. Express 19, 3185-3192 (2011).

[8] A. C. Peacock, J. R. Sparks and N. Healy, “Semiconductor optical fibres: progress and opportunities," Laser \& Photon. Rev. 8, 53 (2014).

[9] C. A. Lin, J. H. Chen, and L. A. Wang, "High-Q silicon microsphere resonators fabricated directly from silicon-cored fibers for whisperinggallery-modes excitation,” IEEE Photon. Technol. Lett., 27,1355-1358 (2015).

[10] L.M. Xiao, M. S. Demokan, W. Jin, Y. P. Wang, and C. L. Zhao, "Fusion Splicing Photonic Crystal Fibers and Conventional Single-Mode Fibers: Microhole Collapse Effect,” J. Lightwave Technol. 25, 3563 (2007).

[11] J. Ma, J. Ju, L. Jin, W. Jin, and D. Wang “Fiber-tip micro-cavity for temperature and transverse load sensing,” Opt. Express, 19, 12418 (2011). 